\title{
MINERALOGIA DE ALGUNS SOLOS DA BACIA SEDIMENTAR DO RECÔNCAVO BAIANO *
}

\author{
Antonio Carlos C.P. Dias \\ J.L.I. DemTtê ***
}

\section{RESUMO}

Foram estudados dez perfis de solos representativos da Bacia Sedimentar do Recôncavo Baiano com o principal objetivo de examinar a mineralogia da fração argila e suas implicações genéticas. Para tanto foram coletados dois perfis de Podzólico Vermelho Amarelo e oito de Grumossois.

Amostras de argila de cada horizonte foram obtidas através de fracionamento de rotina. Tais amostras sofreram pré-tratamento para a eliminação de sais solúveis, matéria orgânica e óxidos de ferro livres. Em seguida foram analisadas qualitativa e quantitativamente.

A caulinita foi o mineral dominante no Podzólico Vermelho Amarelo com teores médios de $55 \%$. Entre os minerais de grade $2: 1$ a mica foi dominante com teores médios de $15 \%$. Ficou evidente a transformação mica-caulinita.

Nos Grumossois a porcentagem dos minerais de grade 2:1 (mica vermiculita e montmorilonita) está em torno de 70 a $80 \%$. Dentre estes a montmorilonita é o mineral dominante. Ficou evidente a transformação montmorilonita caulinita para tais perfis. Mesmo entre os perfis de Grumossois houve diferença de intemperismo. Tais solos foram grupados em: perfis $\mathrm{BS}_{5}$ e $\mathrm{BS}_{8}$ sendo solos com baixo teor de caulinita (teor médio $5 \%$ ); perfis $\mathrm{BS}_{9}$ e $\mathrm{BS}_{10}$ com teor relativamente elevado de caulinita (teor médio $30 \%$ ) e os demais perfis com teores intermediários de caulinita.

\section{INTRODUÇÃO}

O solo é, em última análise, a delgada camada do globo terrestre que suporta e mantém uma parte dos seres vivos. Dentre os seus com-

* Entregue para publicação em 25-3-77.

* Departamento de Solos do CEPEC - Bahia.

*** Departamento de Solos e Geologia da E.S.A. 'Luiz de Queiroz', Piracicaba, SP. 
ponentes estão os minerais secundários constituintes da fração argila. As espécies minerais desta fração dos solos, principalmente das regiões tropicais, são extraordinariamente variáveis devido as multiplas interações dos fatores e processos pedogenéticos.

O conhecimento da composição qualitativa e quantitativa desses componentes do solo é de grande valor, não sć para estudos puramente pedológicos como também constitue um meio eficaz para interpretar ou predizer algumas propriedades do solo de interesse prático, tais como capacidade de troca de cations e de fixação de nutrientes e água, atividade e interação iônica, fornecendo deste subsídios para destino e manejo adequado das terras.

$\mathrm{Na}$ Bacia Sedimentar do Recôncavo Baiano, especificamente em áreas de sedimentos cretácicos, os solos predominantes são Podzólicos Vermelho Amarelo e Grumossois. Estes últimos, vem sendo intensivamente cultivados com cana de açúcar a centenas de anos.

O principal objetivo pretendido neste trabalho foi a análise qualitativa e quantitativa dos minerais da fração argila e o estabelecimento dos estágios de intemperização desses solos, tomando-se como base a composição mineralógica da fração argila dos mesmos.

\section{MATERIAL E MÉTODOS}

\section{Características do meio físico e sclos}

A Bacia Sedimentar do Recôncavo Baiano localiza-se a este do Estado da Bahia com uma área aproximada de $420 \mathrm{~km}^{2}$. Essa bacia foi originada por esforços tencionais e constitui uma das maiores depressões do escudo brasileiro. Os sedimentos são predominantemente de idade cretácica (Silva, 1960) . O clima da região é do tipo Af com uma média de precipitação anual de $1800 \mathrm{~mm}$ e uma temperatura média de $24^{\circ} \mathrm{C}$.

Os solos mais representativos da área de estudo compreendem Podzólicos Vermelho Amarelo e Grumossois. Os primeiros são originários de arenitos e siltitos argilosos que integram diferentes formações geológicas da região. Os grumossois são derivados de siltitos e folhelhos com intercalações de calcário ou cimento calcífico.

Para o presente estudo foram utilizados dez perfis de solos representativos da área sendo dois de Podzólico Vermelho Amarelo e oito 
de grumossois. Os perfis foram identificados com o prefixo BS (Bacia Sedimentar) seguido do número correspondentes a cada um deles. As características morfológicas, físicas e químicas de tais perfis são encontradas em Dias (1971).

\section{Análise da fração argila do solo}

As remoções dos sais solúveis, cations divalentes trocáveis (pelo acetato de sódio a $\mathrm{pH} 5,0$ ) a oxidação da matéria orgânica e eliminação do $\mathrm{MnO}_{\mathrm{z}}$ (pela água oxigenada) a remoção do óxido de ferro livre (pelo ditionito-citrato-bicarbonato de sódio) e as operações de dispersão, fracionamento da fração argila assim como saturação e as operações de aquecimento e glicolação das amostras de argila com $\mathrm{K}^{+}$e com $\mathrm{Mg}+$ foram executados de acordo com as recomendações de Jackson (1965) . As amostras sofreram irradiações em um aparelho Norelco de 2 a $30^{\circ} 2 \theta$ utilizando tubo de cobre e filtro de niquel.

Para a análise quantitativa da fração argila foram utilizados os seguintes métodos: a mica foi determinada tendo-se como base que $10 \%$ de $\mathrm{K}_{\mathrm{z}} \mathrm{O}$ corresponde a $100 \%$ de mica; o material amorfo e caulinita por dissolução seletiva em $\mathrm{KOH}$ 0,5N (Alexiades e Jackson, 1966). Os seguintes minerais foram determinados semi-quantitativamente: vermiculita e montmorilonita (Alexiades e Jackson, 1965) .

\section{RESULTADOS E DISCUSSÃO}

Podzólico Vermelho Amarelo (perfis $B S_{1}$ e $B S_{\mathrm{z}}$ )

O mineral dominante deste solo é a caulinita, com valores entre 50 e $65 \%$ (Quadro 1), decrescendo sensivelmente com a profundidade do solo. A mica (Fig. 1) vem a seguir com teores médios de $14 \%$. $O$ baixo valor da mica nos horizontes $B_{33}$ deistes perfis indica provavelmente um retrabalhamento do material originário. Como trata-se de uma área de sedimentos superpostos e que sofreram alterações tectônicas e posteriores denudações (Silva, 1960) é de se esperar que tais fenômenos causem alterações e mistura do material originário do solo.

Os teores dos demais minerais (montmorilonita, vermiculita e material amorfo) normalmente estão em torno dos $20 \%$ o que vem indicar um intemperismo não muito intenso e semelhante aos valores encontrados por Escobar et alii (1973). 
Quadro 1 - Análise mineralógica da fração argila $(<2 \mu)$ dos perfis estudados

\begin{tabular}{c|c|ccccc}
\hline $\begin{array}{c}\text { Hori- } \\
\text { zontes }\end{array}$ & $\begin{array}{c}\text { Espessura } \\
(\mathrm{cm})\end{array}$ & \multicolumn{6}{c}{\begin{tabular}{c} 
Minerais * $(\%)$ \\
\cline { 3 - 7 }
\end{tabular}} & $\mathrm{C}$ & $\mathrm{A}$ & $\mathrm{Mi}$ & $\mathrm{Mt}$ & $\mathrm{Vm}$ \\
\hline
\end{tabular}

$\begin{array}{lcccccr}\mathrm{Ap} & 0-14 & 64 & 12 & 16 & 3 & 5 \\ \mathrm{~A}_{3} & 14-27 & 64 & 8 & 16 & 5 & 7 \\ \mathrm{~B}_{1} & 27-42 & 65 & 9 & 16 & 5 & 5 \\ \mathrm{~B}_{21} & 42-73 & 63 & 9 & 14 & 5 & 9 \\ \mathrm{~B}_{22} & 73-100 & 62 & 9 & 13 & 6 & 10 \\ \mathrm{~B}_{31} & 100-128 & 58 & 5 & 13 & 6 & 12 \\ \mathrm{~B}_{32} & 128-163 & 57 & 5 & 12 & 7 & 12 \\ \mathrm{~B}_{33} & 163-210 & 51 & 6 & 10 & 8 & 15\end{array}$

Perfil BS

Perfil $\mathbf{B S}_{2}$

$\begin{array}{lcccccc}\mathrm{Ap} & 0-8 & 59 & 5 & 16 & 4 & 10 \\ \mathrm{~A}_{3} & 8-18 & 66 & 4 & 15 & 5 & 10 \\ \mathrm{~B}_{1} & 18-30 & 65 & 4 & 13 & 5 & 13 \\ \mathrm{~B}_{21} & 30-50 & 60 & 4 & 15 & 5 & 12 \\ \mathrm{~B}_{22} & 50-80 & 62 & 4 & 16 & 5 & 12 \\ \mathrm{~B}_{23} & 80-113 & 60 & 4 & 13 & 6 & 17 \\ \mathrm{~B}_{31} & 113-137 & 57 & 3 & 15 & 6 & 10 \\ \mathrm{~B}_{32} & 137-160 & 58 & 3 & 14 & 7 & 12 \\ \mathrm{~B}_{33} & 160-205 & 56 & 3 & 13 & 6 & 15 \\ \mathrm{C} & 205-245 & 57 & 3 & 21 & 7 & 10\end{array}$

Perfil $\mathrm{BS}_{3}$

$\begin{array}{lcrllll}\mathrm{Ap} & 0-30 & 12 & 9 & 15 & 50 & 14 \\ \mathrm{AC}_{1} & 30-48 & 13 & 9 & 13 & 50 & 15 \\ \mathrm{AC}_{2} & 48-80 & 15 & 8 & 12 & 42 & 23 \\ \mathrm{C}_{1} & 80-92 & 11 & 7 & 17 & 52 & 13 \\ \mathrm{C}_{2} & 92-168 & 14 & 9 & 22 & 44 & 1 \\ \mathrm{C} / \mathrm{R} & 168-228 & 5 & 7 & 21 & 47 & 20\end{array}$

Perfis $\mathrm{BS}_{4}$

$\begin{array}{lrlllll}\mathrm{Ap} & 0-14 & 15 & 4 & 20 & 43 & 18 \\ \mathrm{AC} & 14-25 & 16 & 5 & 16 & 46 & 17 \\ \mathrm{C}_{1} & 25-35 & 17 & 3 & 19 & 40 & 21 \\ \mathrm{C}_{2} & 35-75 & 15 & 3 & 23 & 39 & 20 \\ \mathrm{R} & 75-90 & 21 & 2 & 19 & 40 & 18\end{array}$

(Continua) 
Quadro 1 - Continuação

\begin{tabular}{|c|c|c|c|c|c|c|}
\hline \multirow{2}{*}{$\begin{array}{l}\text { Hori- } \\
\text { zontes }\end{array}$} & \multirow{2}{*}{$\begin{array}{l}\text { Espessura } \\
(\mathrm{cm})\end{array}$} & \multicolumn{5}{|c|}{ Minerais * $(\%)$} \\
\hline & & $\mathrm{C}$ & $\mathrm{A}$ & $\mathrm{Mi}$ & $M t$ & $\mathrm{Vm}$ \\
\hline
\end{tabular}

\section{Perfil BS}

$\begin{array}{lrrrrrr}\text { Ap } & 0-20 & 12 & 10 & 21 & 46 & 11 \\ \mathrm{AC} & 20-51 & 5 & 11 & 17 & 55 & 12 \\ \mathrm{C}_{1} & 51-90 & 0 & 7 & 19 & 40 & 34 \\ \mathrm{C}_{2} & 90-125 & 0 & 6 & 23 & 44 & 27\end{array}$

Perfil $\mathrm{BS}_{6}$

$\begin{array}{lcrllll}\mathrm{Ap} & 0-34 & 5 & 6 & 13 & 54 & 22 \\ \mathrm{AC}_{1} & 3-67-67 & 12 & 6 & 11 & 59 & 12 \\ \mathrm{AC}_{2} & 67-94 & 15 & 4 & 13 & 48 & 20 \\ \mathrm{C} & 94-120 & 17 & 3 & 16 & 49 & 15\end{array}$

\section{Perfil $\mathrm{BS}_{7}$}

$\begin{array}{lcrrrrr}\mathrm{Ap} & 0-40 & 9 & 4 & 15 & 45 & 27 \\ \mathrm{AC}_{1} & 40-60 & 12 & 4 & 12 & 47 & 25 \\ \mathrm{AC}_{2} & 60-80 & 12 & 5 & 15 & 50 & 18 \\ \mathrm{C} & 80-110 & 15 & 3 & 18 & 45 & 19\end{array}$

Perfil BS

$\begin{array}{lcccccc}\text { Ap } & 0-50 & 8 & 4 & 17 & 43 & 28 \\ \text { C } & 50-115 & 5 & 5 & 18 & 42 & 30\end{array}$

\section{Perfil $\mathrm{BS}_{9}$}

$\begin{array}{lcccccc}\text { Ap } & 0-20 & 25 & 6 & 17 & 37 & 15 \\ \text { C }_{1} & 20-40 & 26 & 7 & 13 & 37 & 83 \\ \text { IIC }_{2} & 40-70 & 22 & 4 & 18 & 36 & 20 \\ \text { IIR } & 70-101 & 26 & 5 & 21 & 35 & 13\end{array}$

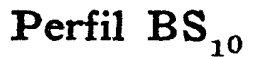

\begin{tabular}{lrlllll}
$\mathrm{Ap}$ & $0-15$ & 36 & 6 & 12 & 30 & 16 \\
$\mathrm{C}_{1}$ & $15-35$ & 35 & 6 & 12 & 37 & 10 \\
$\mathrm{C}_{2}$ & $35-55$ & 32 & 6 & 11 & 38 & 13 \\
$\mathrm{C}_{3}$ & $55-120$ & 37 & 5 & 13 & 37 & 8 \\
$\mathrm{IIC}_{4}$ & $120-165$ & 15 & 6 & 19 & 40 & 20 \\
\hline
\end{tabular}

( $^{*} \mathrm{C}=$ Caulinita $; \mathrm{A}=$ Alofana $\mathrm{Mi}=\mathrm{Mica} ; \mathrm{Mt}=$ Montmorilonita $; \mathrm{Vm}=$ Vermiculita. 
Grumossois (Perfis $B S_{: ;}$a $B S_{10}$ )

De acordo com os valores da caulinita (Quadro 1) é possível grupar os Grumossois em três grupos distintos. Assim é que os perfis BS, e $\mathrm{BS}_{\text {, }}$ formam um grupo de solos com teor relativamente elevado de caulinita (entre 22 e $37 \%$ para os horizontes $C$ ); os perfis $\mathbf{B S}_{5}$ e $\mathbf{B S}_{8}$ com tecres baixos (teor médio de $5 \%$ para os horizontes $\mathrm{C}$ ) e os demais perfis com teores intermediários. O mineral dominante neste solo é a montmorilonita (Fig. 1) cujos valores oscilam entre $30 \%$ e $59 \%$. De uma maneira geral os valores encontrados para a montmorilonita estão relacionados com os de caulinita. Assim é que para o grupo de perfis

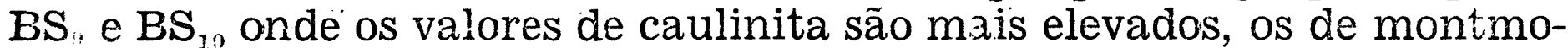
rilonita são os mais baixos. Por outro lado, os perfis $\mathrm{BS}_{5}$ e $\mathbf{B S}_{8}$ possuem baixo valor para a caulinita e elevado valor para a montmorilonita. Tais resultados sugere uma transformação montmorilonita caulinita por dessilicatização (Jackson et alii, 1948) .

O teor de mica, quando comparado com o de montmorilonita, é baixo, em torno de $16 \%$ para a maioria dos perfis. O baixo valor deste mineral se deve a falta de ions específicos necessários para a sua formação, principalmente $\mathrm{K}^{+}$, (Keller, 1964), no material originário, nor-

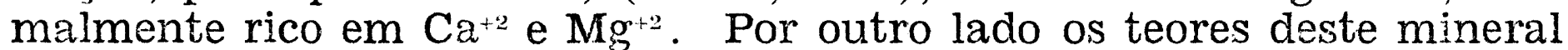
são praticamente constantes de acordo com a profundidade do solo. Tal fato sugere um intemperismo lento para estes solos.

Observando-se a composição mineralógica dos perfis dos Grumossois nota-se que os minerais se distribuem em uma irregular de acordo com a profundidade do solo, isto é, não apresentam uma tendência de aumentar ou diminuir com a profundidade. Este fato pode ser atribuído principalmente a auto-reversão dos solos, isto é, redistribuição do material dos solos ao longo dos perfis através cas fendas produzidas por contração e retração das argilas com o aumento ou diminuição da umidade.

A presença de solos de mineralogia totalmente diferente como é o caso do Podzólico Vermelho Amarelo e do Grumossol ocorrendo na mesma área climática se deve principalmente a dois fatores: natureza do material originário associado com as condições de drenagem do solo. Keller, (1964) salienta que a montmorilonita se forma em um sistema químico caracterizado por uma alta relação Si/Al e com abundância de cálcio, magnésio e ferro e, consequentementa um maixo teor de $\mathrm{H}^{+}$. Completando as afirmações deste autor, Mohr e van Baren (1954) salientam que as condições de má drenagem de um solo é também um fator que favorece a formação destes minerais. O material originário dos grumossois é constituído por folhelhos $\epsilon$ argilitos com cimento calcífero e/ou intercalações de calcário. Tal fato, aliado com as con- 

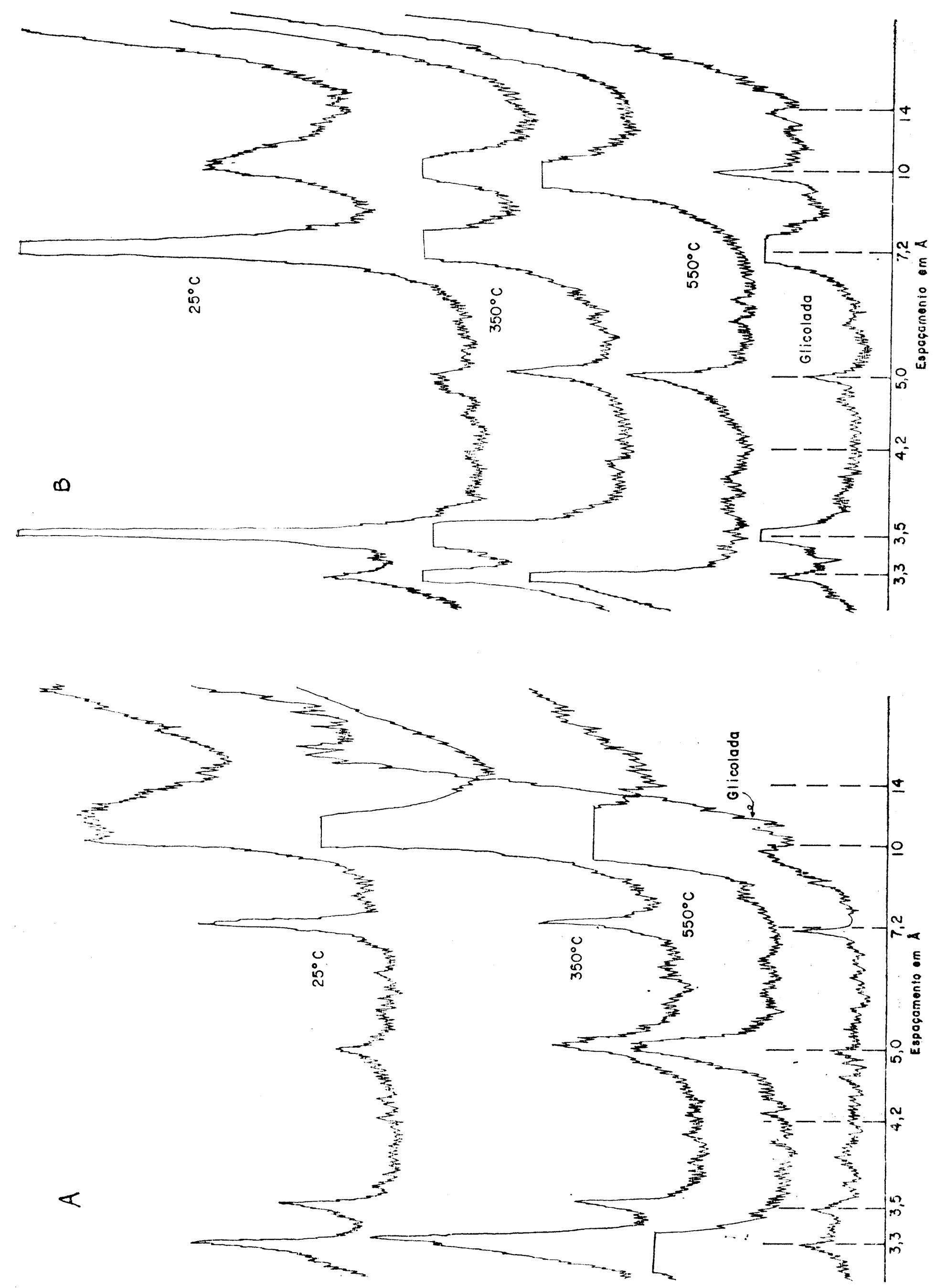

Figura 1 - Difratogramas da fração argila do horizonte AC, perfil BS-3 (A) e do horizonte B22, perfil BS-1 (B) 
dições de drenagem pobre dos Grumossois, o ambiente torna-se favorável à formação de minerais de argila de grade 2:1, principalmente montmorilonita. Por outro lado o materiaì originário do Podzólico Vermelho Amarelo, pobre em cálcio e magnésio, aliado a uma melhor drenagem favorece a formação de caulinita. Neste caso e tomando-se o teor de caulinita como mineral índice pode-se observar que oPodzólico Vermelho Amarelo apresenta uma intemperização moderada enquanto que o Grumossol possue uma intemperização ligeira (Jackson, 1965) .

\section{CONCLUSÕES}

A caulinita foi o mineral dominante no Podzólico Vermelho Amarelo com teores médios de 55\%. Entre os minerais de grade 2:1 a mica foi o dominante com teor médio de $15 \%$. Ficou evidente a transformação mica para caulinita.

- A porcentagem dos minerais de grade 2:1 nos Grumossois está em torno de 70 a $80 \%$. Dentre estes a montmorilonita é o mineral dominante. Ficou evidente a transformação montmorilonita para caulinita nestes solos.

- Houve diferenças de intemperismo entre os perfis de Grumossois. De acordo com o teor de caulinita eles foram grupados em: Perfis $\mathrm{BS}_{5}$ e $\mathrm{BS}_{8}$ em solos com baixo teor de caulinita (teor médio $5 \%$ ); Perfis $\mathrm{BS}_{9}$ e $\mathrm{BS}_{10}$ em solos com teor relativamente elevado de caulinita (teor médio de $30 \%$ ) e os demais perfis com teores intermediários de caulinita.

- A ocorrência tanto do Grumossol como do Podzólico Vermelho Amarelo em uma mesma área climática se deve a diferenças na drenagem e no material de origem.

\section{SUMMARY}

\section{CLAY MINERALOGY FROM SOILS OF RECONCAVO BAIANO, BRAZIL}

Ten soil profiles representative of the region known as "Reconcavo Baiano" (Sedimentary Basin) in Bahia State, were studied with the main objective of collecting information on the mineralogy of their respective clay fraction to determine their weathering sequence. Two of the profiles were taken form a Red-Yellow Podzolic soil and eight from Grumosols (Vertisol). 
Clay fraction were obtained from each horizon of the ten profiles through routine fractionation of the soil sample. These were pre-treated to eliminate soluble salts, organic mather and free iron oxides. The samples were then analysed qualitatively and quantitatively to determine their mineralogical composition.

The results indicated that kaolinite was the dominant clay mineral in the Red-Yellow Podzolic with an average of $55 \%$. Among the 2.1 minerals, mica was the main mineral with $15 \%$. The transformation mica to kaolinite was observed.

The 2:1 minerals (mica, vermiculite and montmorillonite) were dominant minerals in Grumosols (70 to $80 \%)$ and that montmorillonite was the main mineral. The transformation montmorillonite to kaolinite was observed. There was differencces in the weathering in these soils. According to kaolinite amount these profiles were grouped as following :Profiles $\mathrm{BS}_{5}$ and $\mathrm{BS}_{8}$ as soils with low kaolinite amount (average of $5 \%$ ); profiles $\mathrm{BS}_{9}$ and $\mathrm{BS}_{10}$ as relatively high amount of kaolinite (average of $30 \%$ ) and the others profiles as intermediate kaolinite amount.

\section{LITERATURA CITADA}

ALEXIADES, C.A., \& JACKSON, M.L. - Quantitative determination of vermiculite in soils. Soil Sci. Soc. Amer. Proc. $29: 522-527$.

ALEXIADES, C.A. \& JACKON, M.L. - Quantitative clay mineralogical analysis of soils and sediments. Clays and Clay Minerals. 14. 1966.

DIAS, ANTONIO, C.P. C.P. - Mineralogia da fração argilosa de alguns solos da bacia sedimentar do Recôncavo Baiano. Tese de M.S. E.S.A. "Luiz de Queiroz", Piracicaba, SP. 98 p. 1971.

ESCOBAR, E.H., DEMATTE, J.L.I., \& MONIZ, A.C. - Genesis y classiffication de algunos suelos de la cuenca del arroyo Tijuco Preto, Município de Rio das Pedras, São Pablo. I. Analisis mineralogica cuantitativa de la fracion arcilla. Revista de Inv. Agrop. Argentina IX : 89-108, 1972.

JACKSON. M.L., - Weathering sequence of clay-size minerals in soils and sediments: I - Fundamental generalization. J. Phgs. Colloid Chem., 52:1237-60. 1948.

JACKSON, M.L. - Soil Chemical Analysis-Advanced Cource. Madison Wisconsin. 1956.

JACKSON, M.L. - Clay transformation in soil genesis during the quaternary. Soil Sci. $99: 15-22.1965$.

KELLER, W.D. - Processes of origem and alteration of clay minerals. Soil clay mineralogy: A Symposium. Univ. of North Caroline Press. 1964.

MOHR, E.C.J. \& Van BAREN, F.A. - Tropical Soids, Interscience. New York. 1954.

SILVA, T.C. - Repercussão da sedimentação cretária sobre o relevo baiano. Boletim Baiano Geogr. 1:57-65. 1960. 
